\title{
The effect of the look-back period for estimating incidence using administrative data
}

Mira Kim ${ }^{1}$, Kyung-Hee Chae ${ }^{1}$, Youn-Jee Chung ${ }^{2}$, HyeJin Hwang ${ }^{2}$, MinKyung Lee², Hyun-Kyung Kim², Hyun-Hee Cho ${ }^{2}$, Mee-Ran Kim², Chai-Young Jung ${ }^{3}$ and Sukil Kim ${ }^{{ }^{*}}$

\begin{abstract}
Background: The look-back period is needed to define baseline population for estimating incidence. However, short look-back period is known to overestimate incidence of diseases misclassifying prevalent cases to incident cases. The purpose of this study is to evaluate the impact of the various length of look-back period on the observed incidences of uterine leiomyoma, endometriosis and adenomyosis, and to estimate true incidences considering the misclassification errors in the longitudinal administrative data in Korea.
\end{abstract}

Methods: A total of 319,608 women between 15 to 54 years of age in 2002 were selected from Korea National Health Insurance Services (KNHIS) cohort database. In order to minimize misclassification bias incurred when applying various length of look-back period, we used 11 years of claim data to estimate the incidence by equally setting the look-back period to 11 years for each year using prediction model. The association between the year of diagnosis and the number of prevalent cases with the misclassification rates by each look-back period was investigated. Based on the findings, prediction models on the proportion of misclassified incident cases were developed using multiple linear regression.

Results: The proportion of misclassified incident cases of uterine leiomyoma, endometriosis and adenomyosis were $32.8,10.4$ and $13.6 \%$ respectively for the one-year look-back period in 2003 . These numbers decreased to $6.3 \%$ in uterine leiomyoma and $-0.8 \%$ in both endometriosis and adenomyosis using all available look-back periods (11 years) in 2013.

Conclusion: This study demonstrates approaches for estimating incidences considering the different proportion of misclassified cases for various length of look-back period. Although the prediction model used for estimation showed strong R-squared values, follow-up studies are required for validation of the study results.

Keywords: Adenomyosis, Administrative data, Endometriosis, Incidence, Look-back period, Misclassification, Uterine leiomyoma

\footnotetext{
* Correspondence: sikimmd@catholic.ac.kr

'Department of Preventive Medicine, College of Medicine, The Catholic

University of Korea, 222, Banpo-daero, Seocho-gu, Seoul, Republic of Korea

06591

Full list of author information is available at the end of the article
}

(c) The Author(s). 2020 Open Access This article is licensed under a Creative Commons Attribution 4.0 International License, which permits use, sharing, adaptation, distribution and reproduction in any medium or format, as long as you give appropriate credit to the original author(s) and the source, provide a link to the Creative Commons licence, and indicate if changes were made. The images or other third party material in this article are included in the article's Creative Commons licence, unless indicated otherwise in a credit line to the material. If material is not included in the article's Creative Commons licence and your intended use is not permitted by statutory regulation or exceeds the permitted use, you will need to obtain permission directly from the copyright holder. To view a copy of this licence, visit http://creativecommons.org/licenses/by/4.0/. The Creative Commons Public Domain Dedication waiver (http://creativecommons.org/publicdomain/zero/1.0/) applies to the data made available in this article, unless otherwise stated in a credit line to the data. 


\section{Background}

\section{Health insurance claims as a big data}

Administrative data in healthcare primarily refer to the vast medical information available in the form of electronic health records through administrative or health claims data [1]. As the availability of digitized administrative records are increasing, health researchers are able to use these large longitudinal cohort datasets to estimate epidemiologic indicators, such as the incidence and prevalence of various conditions [2-15]. The strengths of this type of large population studies include having a large sample size and avoiding selection or participation bias [16].

The Korean National Health Insurance Service (KNHIS) covers majority of the population as a single payer reimbursing both public and private institutions. All clinics and hospitals submit health insurance claims to the Health Insurance Review and Assessment Service (HIRA) for the claims review each month. The insurance claims include diagnoses (as defined by the International Classification of Diseases 10th revision, ICD-10), demographic information, and medical charges. KNHIS and HIRA share the claims database which represent the entire Korean population and is a major strength in ensuring its applicability for epidemiologic and disease research.

\section{Estimation of incidence rates from administrative data}

Estimating incidence provides a foundation for epidemiologic research, data for resource allocation in health care services, and valuable information for disease prevention. The incidence rate is defined as the ratio of new cases to the total population at risk of the disease. However, the identification of new cases from the administrative data is difficult due to the limited information of patient's disease status prior to the observatory time span of the data.

A common procedure in determining the incident cases is to exclude cases with the respective diagnoses during the look-back period. A long look-back period allows us to identify more accurate incident cases than a short look-back period. But with a long look-back period, valuable data is lost for analyses. A short lookback period, on the other hand, carries the risk of misclassifying prevalent and recurrent cases as incident cases $[17,18]$.

Studies have used various time lengths for look-back period [19-22]. Typically, studies have used 3 to 10 year look-back period [19-22] because a look-back period of less than 3 years can lead to extremely overestimated incidences [23]. However, due to limited data, numerous studies have not considered a look-back period or reported a diagnosis-free interval of 1, 2, or 3 years [24-27]. Additionally, most of studies focused on the estimated the one- year incidence by applying different look-back period [28-30], and there were few studies investigating the incidence trend in longitudinal data. In this study, we intended to investigate the incidence trend considering the increasing look-back period every year in the longitudinal administrative data.

The purposes of this study are to evaluate the impact of various look-back period on the observed incidences of uterine leiomyoma, endometriosis and adenomyosis which are the most common gynecologic diseases in reproductive women and associated with the infertility and adverse pregnancy outcomes [31], and to estimate the true incidences with their trends considering the misclassification error rates using the longitudinal administrative health data in South Korea. While it is advisable to have a sufficiently long look-back period when calculating the incidence using administrative data, we sought a way to minimize data loss.

\section{Methods}

\section{Data source}

We conducted a retrospective population-based cohort study using the National Health Insurance Service-National Sample Cohort (NHIS-NSC) 2002-2013. The data were produced by the KNHIS using a systematic sampling method to generate a representative sample from the target population of 46,605,433 individuals in 2002 . The database is comprised of $1,025,340$ subjects which accounts for approximately $2.2 \%$ of the total eligible Korean population in the year of 2002 who were followed up for 11 years until 2013. The representativeness of the data had been presented elsewhere [32].

It is a semi-dynamically constructed cohort database with individuals that have been followed up to the time of death, emigration, or until the end of the study period and addition of newborn infants included into the database annually [32]. This database includes all medical claims filed from January 2002 to December 2013. More details of the cohort are described elsewhere [32].

\section{Selection of subjects}

Patients in Korea tend to visit several healthcare institutions for any reason, as the patients can access clinics, specialists, and hospitals without restriction. Thus, it is possible for a patient to visit several clinics/hospitals in one day, has multiple diagnostic codes at a time, has multiple claims on the same day in the same clinic/hospital, or has both outpatient treatment and hospital admission on the same day. Therefore, one claim should be selected to define incidence in consideration of all these cases. We set priorities in the following order.

First, priority is given to the claim with the earlier hospital visit date. If there are several patients who visited hospital on same date, inpatient's statement takes priority over outpatient's one. Among several outpatient statements, a statement with a high ranking of diagnosis 
codes is selected in ascending order. If the order of the diagnostic codes is the same, priority is given to that with higher medical costs. Finally, priority is given to the one with earlier billing number. Even though individuals have some gaps of few years between 2002 and 2013 in the record, we considered they are continually insured patients and included in the subject.

A flow chart indicating the number of patients with one of three gynecological diseases is shown in Fig. 1. The population denominator was a total of 319,608 women aged 15-54 who were eligible for the National
Health Insurance in 2002 among 512,082 female individuals from the KNHIS cohort database. Those women were followed up for 11 years until 2012. The incident cases were defined using the standardized codes from the Korean version of the International Classification of Diseases 10th Edition (ICD-10). Cases with diagnostic codes of the target diseases coded in the health insurance claims between 2002 and 2013 regardless of service types were identified; The target diseases of interest were uterine leiomyoma (ICD-10: D25, D25.0, D25.1, D25.2, D25.9), adenomyosis (ICD-10: N80.0), and endometriosis

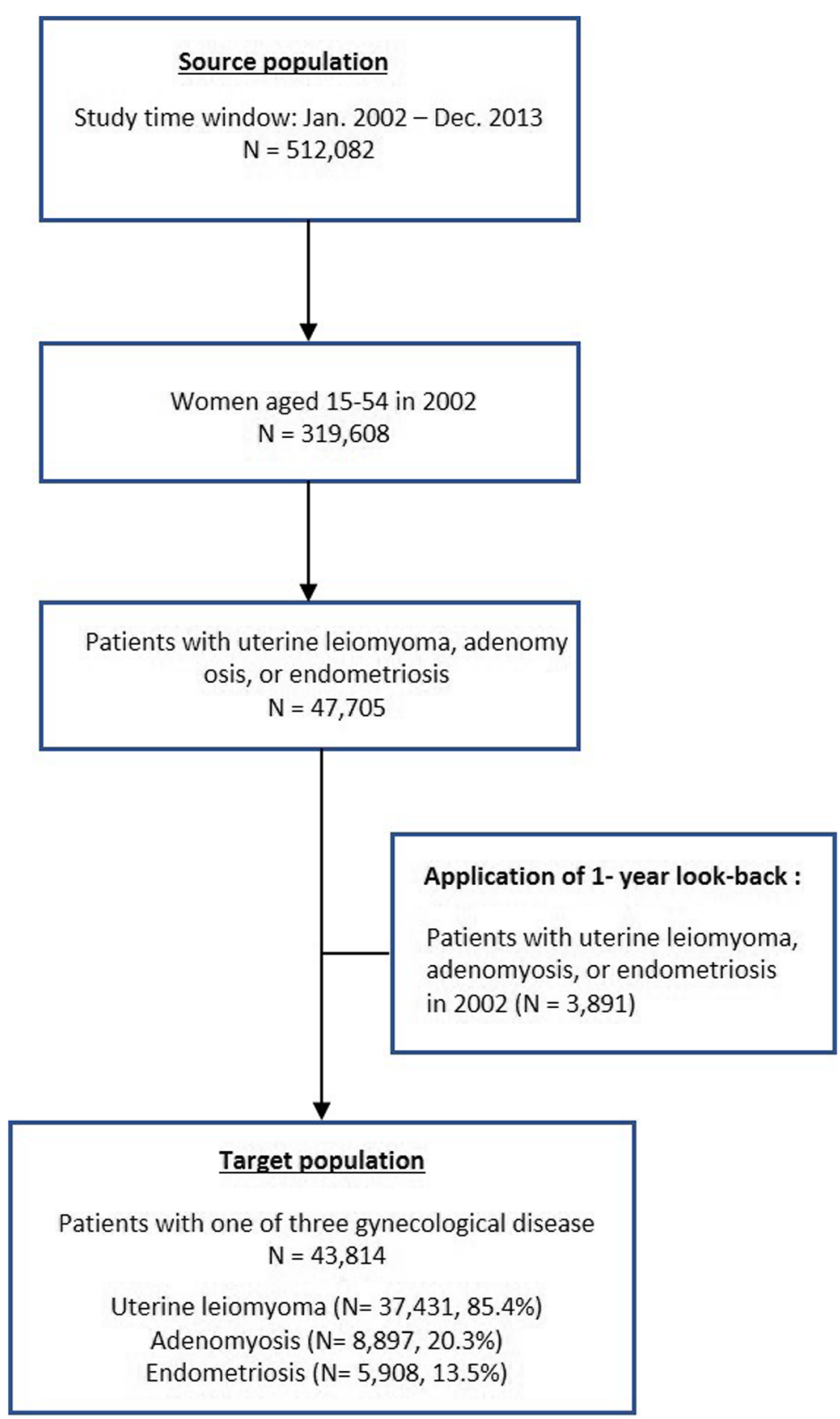

Fig. 1 Flow chart of case identification 
(ICD-10: N80, N80.1, N80.2, N80.3, N80.4, N80.5, N80.6, N80.8, N80.9).

To identify the patients with prior history of the disease, one-year look-back period as of 2003 was applied at the discretion of obstetricians and gynecologists that patients would visit the gynecologists within one year after the onset of diseases. There were 43,814 patients after excluding patients with the target diseases in 2002. Patients who had concurrent diagnoses of uterine leiomyoma, adenomyosis, or endometriosis were counted in each of the targeted disease. Therefore, there were 37, 431 patients with a diagnosis for uterine leiomyoma, 8897 for adenomyosis, and 5908 for endometriosis.

\section{Estimated incidence}

To assess the relationship between the look-back period and the number of misclassified cases, the annual number of patients diagnosed with either uterine leiomyoma, adenomyosis, or endometriosis (prevalent cases) from 2003 to 2013 were determined, and the number of prevalent cases misclassified as incident cases were identified with increasing look-back period by each observation year (Additional File 1).

The association between the year of diagnosis and the number of prevalent cases with the misclassification rates by each look-back period was investigated. Based on the findings, prediction models on the proportion of misclassified incident cases were developed using multiple linear regression. The model of best fit was selected by using the lowest root mean square error (RMSE) or the largest adjusted R-squared value, which are good measures of assessing the accuracy of prediction model. Estimated incidences were calculated using the best prediction model and compared with the observed incidences.

\section{Results}

Misclassification rates of each year by different length of look-back period

The Table 1 shows the number of prevalent cases with uterine leiomyoma in each year. The number of prevalent cases in 2003 was 3092 and continued to increase by year. By 2013, the number of prevalent cases increased to 8348 , which was twice the number of prevalent cases from 2003. Look-back period of each observation year were determined by increasing the look-back period by one year from 2003 (i.e. 2003 had up to 1 one-year look-back period, whereas 2013 had up to 11-year look-back period). With adding more years of look-back period, the proportion of prevalent cases misclassified as incident cases increased.

Table 1 The number of prevalent cases detected by various lengths of look-back period each year (2003-2013) for uterine leiomyoma $\left(\%^{1}\right)$

\begin{tabular}{|c|c|c|c|c|c|c|c|c|c|c|c|c|c|}
\hline \multirow[t]{2}{*}{ Year } & \multirow{2}{*}{$\begin{array}{l}\text { Prevalent } \\
\text { cases }(n)\end{array}$} & \multicolumn{11}{|c|}{ Look-back period (years) } & \multirow{2}{*}{$\begin{array}{l}\text { Estimated } \\
\text { cases }(n, \%)^{2}\end{array}$} \\
\hline & & 1 & 2 & 3 & 4 & 5 & 6 & 7 & 8 & 9 & 10 & 11 & \\
\hline 2003 & 3902 & $\begin{array}{l}785 \\
20.1\end{array}$ & & & & & & & & & & & $\begin{array}{l}1808 \\
46.3\end{array}$ \\
\hline 2004 & 4475 & $\begin{array}{l}1065 \\
23.8\end{array}$ & $\begin{array}{l}1282 \\
28.6\end{array}$ & & & & & & & & & & $\begin{array}{l}2122 \\
47.4\end{array}$ \\
\hline 2005 & 5042 & $\begin{array}{l}1208 \\
24.0\end{array}$ & $\begin{array}{l}1554 \\
30.8\end{array}$ & $\begin{array}{l}1710 \\
33.9\end{array}$ & & & & & & & & & $\begin{array}{l}2445 \\
48.5\end{array}$ \\
\hline 2006 & 5347 & $\begin{array}{l}1417 \\
26.5\end{array}$ & $\begin{array}{l}1840 \\
34.4\end{array}$ & $\begin{array}{l}2047 \\
38.3\end{array}$ & $\begin{array}{l}2151 \\
40.2\end{array}$ & & & & & & & & $\begin{array}{l}2650 \\
49.6\end{array}$ \\
\hline 2007 & 5828 & $\begin{array}{l}1564 \\
26.8\end{array}$ & $\begin{array}{l}2056 \\
35.3\end{array}$ & $\begin{array}{l}2276 \\
39.1\end{array}$ & $\begin{array}{l}2399 \\
41.2\end{array}$ & $\begin{array}{l}2460 \\
42.2\end{array}$ & & & & & & & $\begin{array}{l}2951 \\
50.6\end{array}$ \\
\hline 2008 & 5843 & $\begin{array}{l}1603 \\
27.4\end{array}$ & $\begin{array}{l}2067 \\
35.4\end{array}$ & $\begin{array}{l}2293 \\
39.2\end{array}$ & $\begin{array}{l}2423 \\
41.5\end{array}$ & $\begin{array}{l}2498 \\
42.8\end{array}$ & $\begin{array}{c}2556 \\
43.7\end{array}$ & & & & & & $\begin{array}{l}3021 \\
51.7\end{array}$ \\
\hline 2009 & 6369 & $\begin{array}{l}1795 \\
28.2\end{array}$ & $\begin{array}{l}2397 \\
37.6\end{array}$ & $\begin{array}{l}2726 \\
42.8\end{array}$ & $\begin{array}{l}2895 \\
45.5\end{array}$ & $\begin{array}{l}3008 \\
47.2\end{array}$ & $\begin{array}{c}3090 \\
48.5\end{array}$ & $\begin{array}{c}3135 \\
49.2\end{array}$ & & & & & $\begin{array}{l}3361 \\
52.8\end{array}$ \\
\hline 2010 & 6930 & $\begin{array}{l}2048 \\
29.6\end{array}$ & $\begin{array}{l}2597 \\
37.5\end{array}$ & $\begin{array}{l}2941 \\
42.4\end{array}$ & $\begin{array}{l}3139 \\
45.3\end{array}$ & $\begin{array}{l}3250 \\
46.9\end{array}$ & $\begin{array}{c}3341 \\
48.2\end{array}$ & $\begin{array}{c}3410 \\
49.2\end{array}$ & $\begin{array}{c}3454 \\
49.8\end{array}$ & & & & $\begin{array}{l}3732 \\
53.8\end{array}$ \\
\hline 2011 & 7313 & $\begin{array}{l}2244 \\
30.7\end{array}$ & $\begin{array}{l}2811 \\
38.4\end{array}$ & $\begin{array}{l}3105 \\
42.5\end{array}$ & $\begin{array}{l}3326 \\
45.5\end{array}$ & $\begin{array}{l}3455 \\
47.2\end{array}$ & $\begin{array}{l}3561 \\
48.7\end{array}$ & $\begin{array}{c}3630 \\
49.6\end{array}$ & $\begin{array}{c}3675 \\
50.3\end{array}$ & $\begin{array}{c}3698 \\
50.6\end{array}$ & & & $\begin{array}{l}4016 \\
54.9\end{array}$ \\
\hline 2012 & 8125 & $\begin{array}{l}2542 \\
31.3\end{array}$ & $\begin{array}{l}3253 \\
40.0\end{array}$ & $\begin{array}{l}3632 \\
44.7\end{array}$ & $\begin{array}{l}3829 \\
47.1\end{array}$ & $\begin{array}{l}4013 \\
49.4\end{array}$ & $\begin{array}{c}4130 \\
50.8\end{array}$ & $\begin{array}{c}4210 \\
51.8\end{array}$ & $\begin{array}{r}4257 \\
52.4\end{array}$ & $\begin{array}{c}4303 \\
53.0\end{array}$ & $\begin{array}{c}4338 \\
53.4\end{array}$ & & $\begin{array}{l}4549 \\
56.0\end{array}$ \\
\hline 2013 & 8348 & $\begin{array}{l}2661 \\
31.9\end{array}$ & $\begin{array}{l}3350 \\
40.1\end{array}$ & $\begin{array}{l}3750 \\
44.9\end{array}$ & $\begin{array}{l}3966 \\
47.5\end{array}$ & $\begin{array}{l}4138 \\
49.6\end{array}$ & $\begin{array}{c}4248 \\
50.9\end{array}$ & $\begin{array}{c}4338 \\
52.0\end{array}$ & $\begin{array}{c}4402 \\
52.7\end{array}$ & $\begin{array}{c}4459 \\
53.4\end{array}$ & $\begin{array}{c}4502 \\
53.9\end{array}$ & $\begin{array}{c}4522 \\
54.2\end{array}$ & $\begin{array}{l}4764 \\
57.1\end{array}$ \\
\hline
\end{tabular}

${ }^{1}$ Prevalent cases detected by look-back period divided by prevalent cases

${ }^{2}$ Estimated misclassified cases $(n)$ and the misclassification rate (\%) for 11 years look-back period calculated using the prediction model 
The grey cells at the last column of each observation year show the number of prevalent cases misclassified as incident cases which were discovered by applying the look-back period (Table 1). In 2003 with a one-year look-back period, among a total of 3902 patients with uterine leiomyoma, there were 785 (20.1\%) cases that were misclassified as incident cases. In 2013, however, with 11 years of look-back period among 8348 cases, the misclassified as incident cases increased to 4522 (54.2\%).

Tables 2 and 3 show the proportion of patients diagnosed with adenomyosis and endometriosis and misclassified as incident cases for each look-back period. With a look-back period of 11 years, 733 (41.6\%) patients with adenomyosis and 494 (50.3\%) patients with endometriosis were estimated to have prior history of the disease.

\section{Prediction of the proportions of misclassification}

The year of diagnosis and the number of patients were linearly related with the proportion of misclassification for uterine leiomyoma, adenomyosis and endometriosis, and the look-back period was logarithmically related with the proportion of misclassification (Supplementary Fig. 1, 2 and 3). Using these findings, four prediction models were developed (Table 4). Model A was selected as the model of best fit because it had the smallest RMSE and highest estimated R-squared value. The independent variables were the year of diagnosis and the log-transformed look-back period.

\section{Estimated number of incident cases}

Table 5 shows the number of observed and estimated incident cases per year. The proportions of misclassified cases of uterine leiomyoma, adenomyosis and endometriosis were 32.8, 10.4 and $13.6 \%$, respectively in 2003 with one-year look back period. The proportions of misclassified cases of uterine leiomyoma in 2003 was about 3 times that of adenomyosis and endometriosis. The proportions of misclassified cases decreased to $6.3 \%$ in uterine leiomyoma, $-0.8 \%$ in both adenomyosis and endometriosis in 2013 with 11 years of look-back period.

\section{Discussion}

Administrative health claims database was used to calculate the annual incident cases of uterine leiomyoma, adenomyosis and endometriosis in South Korea (20032013). The proportion of misclassified prevalent cases as incident cases was estimated according to various length of look-back period in years. As the look-back period increased, the proportion of misclassified incident cases decreased. Shorter look-back period incurred incidences with greater proportion of misclassification.

It is difficult to accurately identify new cases in patients diagnosed each year because misclassification bias

Table 2 The number of prevalent cases detected by various lengths of look-back period in each year (2003-2013) for adenomyosis (\% ${ }^{1}$ )

\begin{tabular}{|c|c|c|c|c|c|c|c|c|c|c|c|c|c|}
\hline \multirow[t]{2}{*}{ Year } & \multirow{2}{*}{$\begin{array}{l}\text { Prevalent } \\
\text { cases }(n)\end{array}$} & \multicolumn{11}{|c|}{ Look-back period (years) } & \multirow{2}{*}{$\begin{array}{l}\text { Estimated } \\
\text { cases }(n, \%)^{2}\end{array}$} \\
\hline & & 1 & 2 & 3 & 4 & 5 & 6 & 7 & 8 & 9 & 10 & 11 & \\
\hline 2003 & 650 & $\begin{array}{l}54 \\
8.3\end{array}$ & & & & & & & & & & & $\begin{array}{l}116 \\
17.8\end{array}$ \\
\hline 2004 & 648 & $\begin{array}{l}77 \\
11.9\end{array}$ & $\begin{array}{l}83 \\
12.8\end{array}$ & & & & & & & & & & $\begin{array}{l}130 \\
20.1\end{array}$ \\
\hline 2005 & 849 & $\begin{array}{l}105 \\
12.4\end{array}$ & $\begin{array}{l}130 \\
15.3\end{array}$ & $\begin{array}{l}140 \\
16.5\end{array}$ & & & & & & & & & $\begin{array}{l}191 \\
22.5\end{array}$ \\
\hline 2006 & 823 & $\begin{array}{l}111 \\
13.5\end{array}$ & $\begin{array}{l}143 \\
17.4\end{array}$ & $\begin{array}{l}155 \\
18.8\end{array}$ & $\begin{array}{l}161 \\
19.6\end{array}$ & & & & & & & & $\begin{array}{l}204 \\
24.8\end{array}$ \\
\hline 2007 & 925 & $\begin{array}{l}162 \\
17.5\end{array}$ & $\begin{array}{l}190 \\
20.5\end{array}$ & $\begin{array}{l}202 \\
21.8\end{array}$ & $\begin{array}{l}211 \\
22.8\end{array}$ & $\begin{array}{l}218 \\
23.6\end{array}$ & & & & & & & $\begin{array}{l}251 \\
27.1\end{array}$ \\
\hline 2008 & 1010 & $\begin{array}{l}170 \\
16.8\end{array}$ & $\begin{array}{l}204 \\
20.2\end{array}$ & $\begin{array}{l}225 \\
22.3\end{array}$ & $\begin{array}{l}234 \\
23.2\end{array}$ & $\begin{array}{l}238 \\
23.6\end{array}$ & $\begin{array}{l}240 \\
23.8\end{array}$ & & & & & & $\begin{array}{l}298 \\
29.5\end{array}$ \\
\hline 2009 & 1195 & $\begin{array}{l}241 \\
20.2\end{array}$ & $\begin{array}{l}287 \\
24.0\end{array}$ & $\begin{array}{l}309 \\
25.9\end{array}$ & $\begin{array}{l}332 \\
27.8\end{array}$ & $\begin{array}{l}340 \\
28.5\end{array}$ & $\begin{array}{l}348 \\
29.1\end{array}$ & $\begin{array}{l}352 \\
29.5\end{array}$ & & & & & $\begin{array}{l}380 \\
31.8\end{array}$ \\
\hline 2010 & 1393 & $\begin{array}{l}331 \\
23.8\end{array}$ & $\begin{array}{l}397 \\
28.5\end{array}$ & $\begin{array}{l}422 \\
30.3\end{array}$ & $\begin{array}{l}444 \\
31.9\end{array}$ & $\begin{array}{l}456 \\
32.7\end{array}$ & $\begin{array}{l}464 \\
33.3\end{array}$ & $\begin{array}{l}471 \\
33.8\end{array}$ & $\begin{array}{l}473 \\
34.0\end{array}$ & & & & $\begin{array}{l}476 \\
34.2\end{array}$ \\
\hline 2011 & 1590 & $\begin{array}{l}367 \\
23.1\end{array}$ & $\begin{array}{l}437 \\
27.5\end{array}$ & $\begin{array}{l}471 \\
29.6\end{array}$ & $\begin{array}{l}490 \\
30.8\end{array}$ & $\begin{array}{l}507 \\
31.9\end{array}$ & $\begin{array}{l}519 \\
32.6\end{array}$ & $\begin{array}{l}524 \\
33.0\end{array}$ & $\begin{array}{l}530 \\
33.3\end{array}$ & $\begin{array}{l}530 \\
33.3\end{array}$ & & & $\begin{array}{l}580 \\
36.5\end{array}$ \\
\hline 2012 & 1664 & $\begin{array}{l}444 \\
26.7\end{array}$ & $\begin{array}{l}533 \\
32.0\end{array}$ & $\begin{array}{l}573 \\
34.4\end{array}$ & $\begin{array}{l}600 \\
36.1\end{array}$ & $\begin{array}{l}609 \\
36.6\end{array}$ & $\begin{array}{l}617 \\
37.1\end{array}$ & $\begin{array}{l}622 \\
37.4\end{array}$ & $\begin{array}{l}624 \\
37.5\end{array}$ & $\begin{array}{l}626 \\
37.6\end{array}$ & $\begin{array}{l}628 \\
37.7\end{array}$ & & $\begin{array}{l}646 \\
38.8\end{array}$ \\
\hline 2013 & 1762 & $\begin{array}{l}487 \\
27.6\end{array}$ & $\begin{array}{l}596 \\
33.8\end{array}$ & $\begin{array}{l}646 \\
36.7\end{array}$ & $\begin{array}{l}671 \\
38.1\end{array}$ & $\begin{array}{l}689 \\
39.1\end{array}$ & $\begin{array}{l}699 \\
39.7\end{array}$ & $\begin{array}{l}711 \\
40.4\end{array}$ & $\begin{array}{l}721 \\
40.9\end{array}$ & $\begin{array}{l}724 \\
41.1\end{array}$ & $\begin{array}{l}729 \\
41.4\end{array}$ & $\begin{array}{l}733 \\
41.6\end{array}$ & $\begin{array}{l}725 \\
41.2\end{array}$ \\
\hline
\end{tabular}

${ }^{1}$ Prevalent cases detected by look-back period divided by prevalent cases

${ }^{2}$ Estimated misclassified cases $(n)$ and the misclassification rate (\%) for 11 years look-back period calculated using the prediction model 
Table 3 The number of prevalent cases detected by various lengths of look-back period in each year (2003-2013) for endometriosis (\% ${ }^{1}$ )

\begin{tabular}{|c|c|c|c|c|c|c|c|c|c|c|c|c|c|}
\hline \multirow[t]{2}{*}{ Year } & \multirow{2}{*}{$\begin{array}{l}\text { Prevalent } \\
\text { cases }(n)\end{array}$} & \multicolumn{11}{|c|}{ Look-back period (years) } & \multirow{2}{*}{$\begin{array}{l}\text { Estimated } \\
\text { Cases }(n, \%)\end{array}$} \\
\hline & & 1 & 2 & 3 & 4 & 5 & 6 & 7 & 8 & 9 & 10 & 11 & \\
\hline 2003 & 750 & $\begin{array}{l}127 \\
16.9\end{array}$ & & & & & & & & & & & $\begin{array}{l}212 \\
28.2\end{array}$ \\
\hline 2004 & 770 & $\begin{array}{l}145 \\
18.8\end{array}$ & $\begin{array}{l}164 \\
21.3\end{array}$ & & & & & & & & & & $\begin{array}{l}234 \\
30.4\end{array}$ \\
\hline 2005 & 797 & $\begin{array}{l}168 \\
21.1\end{array}$ & $\begin{array}{l}194 \\
24.3\end{array}$ & $\begin{array}{l}208 \\
26.1\end{array}$ & & & & & & & & & $\begin{array}{l}259 \\
32.5\end{array}$ \\
\hline 2006 & 804 & $\begin{array}{l}194 \\
24.1\end{array}$ & $\begin{array}{l}216 \\
26.9\end{array}$ & $\begin{array}{l}230 \\
28.6\end{array}$ & $\begin{array}{l}236 \\
29.4\end{array}$ & & & & & & & & $\begin{array}{l}279 \\
34.7\end{array}$ \\
\hline 2007 & 738 & $\begin{array}{l}206 \\
27.9\end{array}$ & $\begin{array}{l}245 \\
33.2\end{array}$ & $\begin{array}{l}261 \\
35.4\end{array}$ & $\begin{array}{l}272 \\
36.9\end{array}$ & $\begin{array}{l}275 \\
37.3\end{array}$ & & & & & & & $\begin{array}{l}272 \\
36.9\end{array}$ \\
\hline 2008 & 730 & $\begin{array}{l}219 \\
30.0\end{array}$ & $\begin{array}{l}238 \\
32.6\end{array}$ & $\begin{array}{l}248 \\
34.0\end{array}$ & $\begin{array}{l}253 \\
34.7\end{array}$ & $\begin{array}{l}262 \\
35.9\end{array}$ & $\begin{array}{l}264 \\
36.2\end{array}$ & & & & & & $\begin{array}{l}285 \\
39.0\end{array}$ \\
\hline 2009 & 847 & $\begin{array}{l}231 \\
27.3\end{array}$ & $\begin{array}{l}264 \\
31.2\end{array}$ & $\begin{array}{l}275 \\
32.5\end{array}$ & $\begin{array}{l}290 \\
34.2\end{array}$ & $\begin{array}{l}304 \\
35.9\end{array}$ & $\begin{array}{l}309 \\
36.5\end{array}$ & $\begin{array}{l}311 \\
36.7\end{array}$ & & & & & $\begin{array}{l}349 \\
41.2\end{array}$ \\
\hline 2010 & 959 & $\begin{array}{l}309 \\
32.2\end{array}$ & $\begin{array}{l}348 \\
36.3\end{array}$ & $\begin{array}{l}375 \\
39.1\end{array}$ & $\begin{array}{l}397 \\
41.4\end{array}$ & $\begin{array}{l}406 \\
42.3\end{array}$ & $\begin{array}{l}410 \\
42.8\end{array}$ & $\begin{array}{l}418 \\
43.6\end{array}$ & $\begin{array}{l}421 \\
43.9\end{array}$ & & & & $\begin{array}{l}416 \\
43.4\end{array}$ \\
\hline 2011 & 965 & $\begin{array}{l}360 \\
37.3\end{array}$ & $\begin{array}{l}389 \\
40.3\end{array}$ & $\begin{array}{l}403 \\
41.8\end{array}$ & $\begin{array}{l}417 \\
43.2\end{array}$ & $\begin{array}{l}426 \\
44.1\end{array}$ & $\begin{array}{l}437 \\
45.3\end{array}$ & $\begin{array}{l}442 \\
45.8\end{array}$ & $\begin{array}{l}449 \\
46.5\end{array}$ & $\begin{array}{l}450 \\
46.6\end{array}$ & & & $\begin{array}{l}439 \\
45.5\end{array}$ \\
\hline 2012 & 957 & $\begin{array}{l}350 \\
36.6\end{array}$ & $\begin{array}{l}385 \\
40.2\end{array}$ & $\begin{array}{l}404 \\
42.2\end{array}$ & $\begin{array}{l}411 \\
42.9\end{array}$ & $\begin{array}{l}423 \\
44.2\end{array}$ & $\begin{array}{l}429 \\
44.8\end{array}$ & $\begin{array}{l}431 \\
45.0\end{array}$ & $\begin{array}{l}433 \\
45.2\end{array}$ & $\begin{array}{l}439 \\
45.9\end{array}$ & $\begin{array}{l}442 \\
46.2\end{array}$ & & $\begin{array}{l}457 \\
47.7\end{array}$ \\
\hline 2013 & 983 & $\begin{array}{l}366 \\
37.2\end{array}$ & $\begin{array}{l}422 \\
42.9\end{array}$ & $\begin{array}{l}445 \\
45.3\end{array}$ & $\begin{array}{l}452 \\
46.0\end{array}$ & $\begin{array}{l}456 \\
46.4\end{array}$ & $\begin{array}{l}464 \\
47.2\end{array}$ & $\begin{array}{l}471 \\
47.9\end{array}$ & $\begin{array}{l}480 \\
48.8\end{array}$ & $\begin{array}{l}489 \\
49.7\end{array}$ & $\begin{array}{l}493 \\
50.2\end{array}$ & $\begin{array}{l}494 \\
50.3\end{array}$ & $\begin{array}{l}490 \\
49.9\end{array}$ \\
\hline
\end{tabular}

${ }^{1}$ Prevalent cases detected by look-back period divided by prevalent cases

${ }^{2}$ Estimated misclassified cases $(n)$ and the misclassification rate (\%) for 11 years look-back period calculated using the prediction model

Table 4 Comparison of the prediction models by RMSE and estimated $\mathrm{R}^{2}$

\begin{tabular}{|c|c|c|c|c|c|c|c|}
\hline \multirow[t]{2}{*}{ Model } & \multicolumn{2}{|c|}{ Independent variable } & \multirow[t]{2}{*}{ intercept } & \multicolumn{2}{|c|}{ Regression coefficient $^{1}$} & \multirow[t]{2}{*}{ RMSE } & \multirow[t]{2}{*}{$\operatorname{Adj} R^{2}$} \\
\hline & $\overline{X_{1}}$ & $x_{2}$ & & $\overline{B_{1}}$ & $B_{2}$ & & \\
\hline \multicolumn{8}{|c|}{ Uterine leiomyoma } \\
\hline A & Ln (Look-back) & Year & 1 & 0.0967 & 0.01072 & 0.01297 & 0.9788 \\
\hline B & Ln (Look-back) & Patients size & 0.1385 & 0.09757 & 0.00002347 & 0.01387 & 0.9757 \\
\hline$C$ & Look-back & Year & -22.6134 & 0.0233 & 0.01141 & 0.03295 & 0.8632 \\
\hline $\mathrm{D}$ & Look-back & Patients size & 0.14922 & 0.02358 & 0.00002459 & 0.03375 & 0.8565 \\
\hline \multicolumn{8}{|c|}{ Adenomyosis } \\
\hline A & Ln (Look-back) & Year & -46.7434 & 0.0464 & 0.02337 & 0.01371 & 0.9745 \\
\hline B & Ln (Look-back) & Patients size & -0.00698 & 0.04887 & 0.00017117 & 0.01637 & 0.9636 \\
\hline C & Look-back & Year & -47.0381 & 0.01154 & 0.02352 & 0.01877 & 0.9522 \\
\hline D & Look-back & Patients size & 0.000494 & 0.01203 & 0.00017177 & 0.02195 & 0.9346 \\
\hline \multicolumn{8}{|c|}{ Endometriosis } \\
\hline A & Ln (Look-back) & Year & 1.83882 & 0.0034 & 0.00091597 & 0.01762 & 0.9549 \\
\hline B & Ln (Look-back) & Patients size & -0.12183 & 0.0609 & 0.00047936 & 0.03409 & 0.8312 \\
\hline$C$ & Look-back & Year & -43.6181 & 0.01176 & 0.02187 & 0.02229 & 0.9278 \\
\hline D & Look-back & Patients size & -0.10688 & 0.01539 & 0.0004722 & 0.03793 & 0.7911 \\
\hline
\end{tabular}

${ }^{1} B 1$ and $B 2$ are the regression coefficients of independent variable $X_{1}$ and $X_{2}$ 
Table 5 The proportions of misclassified between observed incident cases and estimated incident cases

\begin{tabular}{|c|c|c|c|c|c|c|c|c|c|c|c|}
\hline \multirow[t]{2}{*}{ Incidence } & \multicolumn{11}{|c|}{ Look-back period (years) } \\
\hline & $2003(1)$ & $2004(2)$ & $2005(3)$ & $2006(4)$ & 2007 (5) & $2008(6)$ & $2009(7)$ & $2010(8)$ & $2011(9)$ & $2012(10)$ & $2013(11)$ \\
\hline \multicolumn{12}{|l|}{ Uterine leiomyoma } \\
\hline Observed (n) & 3117 & 3193 & 3332 & 3196 & 3368 & 3287 & 3234 & 3476 & 3615 & 3787 & 3826 \\
\hline Estimated $(n)$ & 2094 & 2353 & 2597 & 2697 & 2877 & 2822 & 3008 & 3198 & 3297 & 3576 & 3584 \\
\hline Proportions of misclassified (\%) & 32.8 & 26.3 & 22.1 & 15.6 & 14.6 & 14.1 & 7.0 & 8.0 & 8.8 & 5.6 & 6.3 \\
\hline \multicolumn{12}{|l|}{ Adenomyosis } \\
\hline Observed & 596 & 565 & 709 & 662 & 707 & 770 & 843 & 920 & 1060 & 1036 & 1029 \\
\hline Estimated & 534 & 518 & 658 & 619 & 674 & 712 & 815 & 917 & 1010 & 1018 & 1037 \\
\hline Proportions of misclassified (\%) & 10.4 & 8.3 & 7.2 & 6.5 & 4.7 & 7.5 & 3.3 & 0.3 & 4.7 & 1.7 & -0.8 \\
\hline \multicolumn{12}{|l|}{ Endometriosis } \\
\hline Observed & 623 & 606 & 589 & 568 & 463 & 466 & 536 & 538 & 515 & 515 & 489 \\
\hline Estimated & 538 & 536 & 538 & 525 & 466 & 445 & 498 & 543 & 526 & 500 & 493 \\
\hline Proportions of misclassified (\%) & 13.6 & 11.6 & 8.7 & 7.6 & -0.6 & 4.5 & 7.1 & -0.9 & -2.1 & 2.9 & -0.8 \\
\hline
\end{tabular}

exists in which the prevalent case is considered as an incidence case according to look-back period changing every year during the research period. Thus, to minimize this systematic error, we used 11 years of claim data to estimate the incidence by equally setting the look-back period to 11 years for each year using prediction model.

\section{Optimal look-back period for annual incidence}

As mentioned in the Abbas's study, the optimal lookback period for annual incidence while minimizing the rate of misclassification depended on the nature and the stage of the respective diseases [23]. In uterine leiomyoma and adenomyosis, the proportion of misclassified cases decreased by about $50 \%$ when the look-back period increased from 6 years to 7 years, and in endometriosis, it decreased by about $10 \%$ when the look-back period increased from 7 years to 8 years. The proportion of misclassified cases of endometriosis in 2007 is -0.6 which is considerably smaller than 7.6, the rate of previous year. Therefore, disease-specific look-back period required at least 7 years for uterine leiomyoma and adenomyosis, and 8 years for endometriosis.

The extent of misclassification varies by diseases even though the same length of look-back period was applied. In 2003 with one-year look-back period, the proportion of misclassification for uterine leiomyoma was $32.8 \%$, while for adenomyosis and endometriosis were 10.4 and $13.6 \%$, respectively. Similarly, in the 11 years of lookback period in 2013, the proportion of misclassification for uterine leiomyoma was $6.3 \%$ and $-0.8 \%$ for adenomyosis and endometriosis, which is negligible.

Incidences can be affected by external effect. The number of endometriosis patients significantly decreased in 2007, and thereafter did not increase. One possible reason is that the HIRA has strengthened coding requirement to use full digit detail codes in 2006. Subsequently, the number of endometriosis patients with N80 might be redistributed to N80.0 for adenomyosis and N80.1 to N80.9 for the endometriosis. The estimated number of incident cases of the disease in 2013 should be interpreted with caution. When the estimated incidence is lower than the observed incidence, the observed incidence should be used instead of the estimated incidence for the practical use.

According to the Organization for Economic Cooperation and Development (OECD) statistics in 2018, the annual number of outpatient visits per capita in Korea in 2016 was 17.0 which is the highest among OECD countries and 2.5 times more than the OECD average (6.9) [33]. As such, the same duration of lookback period using administrative health data in Korea is estimated to have an increase in the proportion of misclassification than other OECD countries.

\section{Strengths and limitations}

The strengths of this study include large sample size and long observation period of 12 years. This increases the accuracy for calculating the incidences and proportion of misclassifications. However, the study has several limitations.

In the regression model for estimating the number of incident cases, a linear function for the observation year and a $\log$ function for the look-back was used. There were 11 data points for the one-year look-back, but only one point for the 11-year look-back. Although the prediction model had a good RMSE and R-squared, the model was based on uneven distribution of the observed data points may adversely affected the fit of the model.

The study has inherent limitations as this study was based on secondary data analyses of the NHIS cohort 
database. We could not definitely confirm the diagnosis codes for every single patient in the database since the diagnostic code of the claim data alone cannot guarantee the accuracy of the diagnosis [34]. According to Park et al. [35], about $70 \%$ of primary diagnosis codes concurred with medical records. Issues concerning studies involving administrative data are well described in Mazzali, C. and and P. Duca's study [36]. When the cases were confirmed by prescription codes and procedure code in addition to the diagnostic codes, the incidences would be lower than this study results. Lastly, asymptomatic and/or undiagnosed patients cannot be detected using the health claims data. This would decrease the proportion of the true incident cases of the diseases.

\section{Conclusion}

Using the NHIS administrative heath database, various length of the look-back period was applied to estimate the incidences of uterine leiomyoma, adenomyosis, and endometriosis and determine the different proportion of misclassification errors for each look-back period. The prediction model was used to adjust the misclassification errors that occur when calculating incidence trend derived from longitudinal administrative data. Although the prediction model used for estimation showed strong $\mathrm{R}$-squared values, follow-up studies are required for validation of the study results.

In the longitudinal data, the look-back period applied for incidence estimation generated different misclassification errors for each look-back period. We proposed a method to adjust the misclassification errors when calculating the incidence using administrative data. Even though we focused on the three gynecological disease in this study, the approaches presented in this study are applicable to other diseases as well.

\section{Supplementary information}

Supplementary information accompanies this paper at https://doi.org/10. 1186/s12913-020-5016-y.

Additional file 1. A detailed description of model construction.

Additional file 2: Supplementary Figure S1. The number of prevalent cases and misclassification rate detected by various lengths of the lookback period per year between 2003 and 2013 forwomen with uterine leiomyoma.

Additional file 3: Supplementary Figure S2. The number of prevalent cases and misclassification rate detected by various lengths of the lookback period per year between 2003 and 2013 for women with adenomyosis.

Additional file 4: Supplementary Figure S3. The number of prevalent cases and misclassification rate detected by various lengths of the lookback period per year between 2003 and 2013 for women with endometriosis.

\section{Abbreviations}

HIRA: Health Insurance Review and Assessment Service; ICD-10: International Classification of Diseases 10th revision; KNHIS: Korean National Health
Insurance Service; NHIS-NSC: National Health Insurance Service-National Sample Cohort; OECD: Organization for Economic Co-operation and Development; RSME: Root mean square error

\section{Acknowledgements}

Not applicable.

\section{Prior presentation}

The part of this work presented at the International Population Data Linkage Conference in Banff, Canada 2018. https://ijpds.org/article/view/883

\section{Authors' contributions}

MK and SK designed the study, reviewed related paper, built the research hypothesis and wrote a draft of manuscript. KHC was involved in data acquisition and performed the analysis. YJC, HJH, MKL, HKK, HHC, MRK and CYJ provided important comments on the research design and interpretation of the results. All authors read and approved the final manuscript.

\section{Funding}

This work was funded by the Korea Health Industry Development Institute Research Program, Healthcare Big Data R\&D Support grant to MRK [HI16C1107]. MRK as a co-author of this paper, provided intellectual and editorial input.

\section{Availability of data and materials}

The data that support the findings of this study are not publicly available. Data are however available from the Korea National Health Insurance Service (KNHIS) upon request and with permission of KNHIS.

\section{Ethics approval and consent to participate}

Ethics approval for this study was obtained from the Institutional Review Board Seoul St. Mary's Hospital (KIRB-0E513-001). The IRB approved that formal consent was not necessary for this study because patient records/ information was anonymized and de-identified prior to analysis.

Consent for publication

Not applicable.

\section{Competing interests}

The authors declare that they have no competing interests.

\section{Author details}

${ }^{1}$ Department of Preventive Medicine, College of Medicine, The Catholic University of Korea, 222, Banpo-daero, Seocho-gu, Seoul, Republic of Korea 06591. ${ }^{2}$ Department of Obstetrics and Gynecology, College of Medicine, The Catholic University of Korea, Seoul, Republic of Korea. ${ }^{3}$ Biomedical Research Institute, Inha University Hospital, Incheon, Republic of Korea.

Received: 6 June 2019 Accepted: 20 February 2020

Published online: 04 March 2020

\section{References}

1. Trifiro G, Sultana J, Bate A. From big data to smart data for Pharmacovigilance: the role of healthcare databases and other emerging sources. Drug Saf. 2017.

2. Gothe $\mathrm{H}$, et al. The use of administrative data to determine prevalence and incidence of Copd: a systematic review. Value Health. 2016;19(7):A368-9.

3. Widdifield J, et al. Development and validation of an administrative data algorithm to estimate the disease burden and epidemiology of multiple sclerosis in Ontario, Canada. Multiple Scler. 2015;21(8):1045.

4. Ng, R., et al. Brain disorders in Ontario: prevalence, incidence and costs from health administrative data. Institute for Clinical Evaluative Sciences: Toronto, Ontario, 2015

5. Nigwekar SU, et al. Quantifying a rare disease in administrative data: the example of calciphylaxis. J Gen Intern Med. 2014;29(3):724-31.

6. Benchimol El, et al. Validation of international algorithms to identify adults with inflammatory bowel disease in health administrative data from Ontario, Canada. J Clin Epidemiol. 2014;67(8):887-96.

7. Ward MM. Estimating disease prevalence and incidence using administrative data: some assembly required. J Rheumatol. 2013;40(8):1241-3. 
8. Marrie RA, et al. The incidence and prevalence of thyroid disease do not differ in the multiple sclerosis and general populations: a validation study using administrative data. Neuroepidemiology. 2012;39(2):135-42.

9. Floyd JS, et al. Use of administrative data to estimate the incidence of statin-related rhabdomyolysis. JAMA. 2012;307(15):1580-2.

10. Benchimol $\mathrm{El}$, et al. Increasing incidence of paediatric inflammatory bowel disease in Ontario, Canada: evidence from health administrative data. Gut. 2009;58(11):1490-7.

11. Mirkin D, Murphy-Barron C, Iwasaki K. Actuarial analysis of private payer administrative claims data for women with endometriosis. J Manag Care Pharm. 2007;13(3):262-72.

12. Bernatsky $\mathrm{S}$, et al. A population-based assessment of systemic lupus erythematosus incidence and prevalence-results and implications of using administrative data for epidemiological studies. Rheumatology. 2007;46(12): 1814-8.

13. Segal J, Powe N. Prevalence of immune thrombocytopenia: analyses of administrative data. J Thromb Haemost. 2006:4(11):2377-83.

14. Hux JE, et al. Diabetes in Ontario: determination of prevalence and incidence using a validated administrative data algorithm. Diabetes Care. 2002;25(3):512-6.

15. Hamad R, et al. Using "big data" to capture overall health status: properties and predictive value of a claims-based health risk score. PLoS One. 2015; 10(5):e0126054.

16. Chen YC, et al. Reduced access to database. A publicly available database accelerates academic production. BMJ. 2011:342:d637.

17. Czwikla J, Jobski K, Schink T. The impact of the lookback period and definition of confirmatory events on the identification of incident cancer cases in administrative data. BMC Med Res Methodol. 2017;17(1):122.

18. Sulo $\mathrm{G}$, et al. Effect of the Lookback Period's length used to identify incident acute myocardial infarction on the observed trends on incidence rates and survival: cardiovascular disease in Norway project. Circ Cardiovasc Qual Outcomes. 2015;8(4):376-82.

19. Yeh RW, et al. Population trends in the incidence and outcomes of acute myocardial infarction. N Engl J Med. 2010;362(23):2155-65.

20. Koopman C, et al. Population trends and inequalities in incidence and short-term outcome of acute myocardial infarction between 1998 and 2007. Int J Cardiol. 2013;168(2):993-8.

21. Salomaa $\mathrm{V}$, et al. Use of secondary preventive medications after the first attack of acute coronary syndrome. Eur J Cardiovasc Prev Rehabil. 2007; 14(3):386-91.

22. Lin JC, Shau WY, Lai MS. Sex- and age-specific prevalence and incidence rates of sight-threatening diabetic retinopathy in Taiwan. JAMA Ophthalmol. 2014;132(8):922-8.

23. Abbas $\mathrm{S}$, et al. Estimation of disease incidence in claims data dependent on the length of follow-up: a methodological approach. Health Serv Res. 2012; 47(2):746-55.

24. Boehme MW, et al. Prevalence, incidence and concomitant co-morbidities of type 2 diabetes mellitus in South Western Germany--a retrospective cohort and case control study in claims data of a large statutory health insurance. BMC Public Health. 2015;15:855.

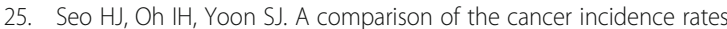
between the national cancer registry and insurance claims data in Korea. Asian Pac J Cancer Prev. 2012;13(12):6163-8.

26. $\mathrm{Kim} \mathrm{H}$, et al. Estimating epilepsy incidence and prevalence in the US pediatric population using Nationwide health insurance claims data. J Child Neurol. 2016;31(6):743-9.

27. Linsell $L$, et al. Prevalence and incidence of adults consulting for shoulder conditions in UK primary care; patterns of diagnosis and referral. Rheumatology (Oxford). 2006:45(2):215-21.

28. Schmedt $\mathrm{N}$, et al. Incidence of multiple sclerosis in Germany: a cohort study applying different case definitions based on claims data. Neuroepidemiology. 2017:49(3-4):91-8.

29. Smolina K, et al. Incidence and 30-day case fatality for acute myocardial infarction in England in 2010: national-linked database study. Eur J Public Health. 2012;22(6):848-53.

30. Worthington $\mathrm{JM}$, et al. Differentiating incident from recurrent stroke using administrative data: the impact of varying lengths of look-Back periods on the risk of misclassification. Neuroepidemiology. 2017;48(3-4):111-8.

31. Wise LA, Laughlin-Tommaso SK. Epidemiology of uterine fibroids: from menarche to menopause. Clin Obstet Gynecol. 2016:59(1):2-24.
32. Lee J, et al. Cohort profile: the National Health Insurance Service-National Sample Cohort (NHIS-NSC), South Korea. Int J Epidemiol. 2017:46(2):e15.

33. OECD. OECD Health Statistics 2018. [cited 2018 Sep. 3]; Available from: http://www.oecd.org/els/health-systems/.

34. Sohn $\mathrm{S}$, et al. A nationwide epidemiological study of newly diagnosed spine metastasis in the adult Korean population. Spine J. 2016;16(8):937-45.

35. Park BJ, Park PK, sung KH. Validity of diagnosis code on National Health Insurance Claim Database. Seoul: Seoul National University School of Medicine; 2003

36. Mazzali C, Duca P. Use of administrative data in healthcare research. Intern Emerg Med. 2015;10(4):517-24.

\section{Publisher's Note}

Springer Nature remains neutral with regard to jurisdictional claims in published maps and institutional affiliations.
Ready to submit your research? Choose BMC and benefit from:

- fast, convenient online submission

- thorough peer review by experienced researchers in your field

- rapid publication on acceptance

- support for research data, including large and complex data types

- gold Open Access which fosters wider collaboration and increased citations

- maximum visibility for your research: over $100 \mathrm{M}$ website views per year

At $\mathrm{BMC}$, research is always in progress.

Learn more biomedcentral.com/submissions 